\title{
Trends in the incidence and prevalence of Alzheimer's disease, dementia, and cognitive impairment in the United States
}

\author{
Walter A. Rocca ${ }^{\mathrm{a}, \mathrm{b}, *}$, Ronald C. Petersen ${ }^{\mathrm{a}, \mathrm{b}, \mathrm{c}}$, David S. Knopman ${ }^{\mathrm{b}, \mathrm{c}}$, Liesi E. Hebert ${ }^{\mathrm{d}}$, \\ Denis A. Evans ${ }^{\mathrm{d}, \mathrm{e}}$, Kathleen S. Hall ${ }^{\mathrm{f}}$, Sujuan $\mathrm{Gao}^{\mathrm{g}}$, Frederick W. Unverzagt ${ }^{\mathrm{f}}$, \\ Kenneth M. Langa ${ }^{\mathrm{h}, \mathrm{i}, \mathrm{j}}$, Eric B. Larson ${ }^{\mathrm{k}}$, Lon R. White ${ }^{\mathrm{l}, \mathrm{m}, \mathrm{n}}$ \\ ${ }^{a}$ Division of Epidemiology, Department of Health Sciences Research, College of Medicine, Mayo Clinic, Rochester, MN, USA \\ ${ }^{b}$ Department of Neurology, College of Medicine, Mayo Clinic, Rochester, MN, USA \\ 'Mayo Alzheimer's Disease Research Center, Mayo Clinic, Rochester, MN, USA \\ ${ }^{d}$ Rush Institute for Healthy Aging, Rush University Medical Center, Chicago, IL, USA \\ ${ }^{e}$ Department of Internal Medicine, Rush University Medical Center, Chicago, IL, USA \\ ${ }^{f}$ Department of Psychiatry, School of Medicine, Indiana University, Indianapolis, IN, USA \\ ${ }^{g}$ Department of Biostatistics, School of Medicine, Indiana University, Indianapolis, IN, USA \\ ${ }^{h}$ Division of General Medicine, Department of Medicine, School of Medicine, University of Michigan, Ann Arbor, MI, USA \\ ${ }^{i}$ Veterans Affairs Center for Practice Management and Outcomes Research, Ann Arbor, MI, USA \\ ${ }^{j}$ Institute for Social Research, University of Michigan, Ann Arbor, MI, USA \\ ${ }^{k}$ Group Health Research Institute, Seattle, WA, USA \\ 'Kuakini Medical Center, Honolulu, HI, USA \\ ${ }^{m}$ Department of Biology, Chaminade University, Honolulu HI, USA \\ ${ }^{n}$ Department of Pathology, School of Medicine, University of Washington, Seattle, WA, USA
}

Abstract

Declines in heart disease and stroke mortality rates are conventionally attributed to reductions in cigarette smoking, recognition and treatment of hypertension and diabetes, effective medications to improve serum lipid levels and to reduce clot formation, and general lifestyle improvements. Recent evidence implicates these and other cerebrovascular factors in the development of a substantial proportion of dementia cases. Analyses were undertaken to determine whether corresponding declines in age-specific prevalence and incidence rates for dementia and cognitive impairment have occurred in recent years. Data spanning 1 or 2 decades were examined from community-based epidemiological studies in Minnesota, Illinois, and Indiana, and from the Health and Retirement Study, which is a national survey. Although some decline was observed in the Minnesota cohort, no statistically significant trends were apparent in the community studies. A significant reduction in cognitive impairment measured by neuropsychological testing was identified in the national survey. Cautious optimism appears justified.

(C) 2011 The Alzheimer's Association. All rights reserved.

Keywords:

Alzheimer's disease; Dementia; Cognitive impairment; Prevalence; Incidence; Time trends

\section{Introduction}

Life expectancy across the globe has increased dramatically over the past century [1]. However, this salutary trend also has a negative aspect, as the common chronic diseases

All of the authors contributed equally to this article.

*Corresponding author. Tel.: +1 507284 3568; Fax: +1 5072841516.

E-mail address: rocca@mayo.edu of older people are becoming much more prevalent. The number of people affected by Alzheimer's disease (AD) was 26.6 million worldwide in 2006, and it was estimated that $\$ 156$ billion is spent annually to care for patients with dementia worldwide [2]. By 2050, the prevalence is expected to quadruple, so that one in 85 persons will be living with the disease [3], and $43.0 \%$ of them are expected to need a high level of care (e.g., a nursing home). Although the prevalence of dementia and its associated disability increases exponentially with age [4-6], the focus of research 
has recently shifted toward younger persons and the early stages of cognitive decline and mild cognitive impairment. The aim is now to discover pathogenetic mechanisms underlying dementia and to delay the conversion of cognitive decline and mild cognitive impairment to full dementia [7]. Indeed, if interventions could delay disease onset or progression by as little as 1 year, nearly 9.2 million fewer AD patients would be expected by the year 2050 [3].

Dementia and AD represent a significant public health challenge for U.S. society, which is only likely to increase as the population ages. For example, there has been a large increase in diagnosis of AD on U.S. death certificates in the last 10 years. However, it is important to separate increases in death certificate diagnoses because of growing awareness of the disease from increases caused by the changes in disease occurrence or mortality. Epidemiologists have attempted to uncover a wide range of risk and protective factors for dementia. Advocacy and public health organizations have also made efforts over the last 3 decades to increase the awareness of dementia and its known risk factors among both the public and practicing physicians. Thus far, these efforts have likely altered the risk of dementia only moderately, if at all. Society's investment in dementia research must eventually be justified by progress made toward reducing the incidence of dementia or at least its associated disability.

On the basis of time trends in potential risk and protective factors for cognitive impairment, there are good reasons to expect a decline in the incidence rates of cognitive impairment over time. However, there are also some trends in cardiovascular risk factors that would suggest an expected increase. A cluster of demographic, lifestyle, and medical factors have been identified which seem to alter the risk of developing dementia [8,9]. Among these, vascular risk factors are prominent, including hypertension and diabetes $[10,11]$. Some decline in the risk of dementia might also be expected because cardiovascular disease and cerebrovascular disease contribute to the risk of dementia and there has been a substantial decline in the incidence of stroke in the last 50 years. Alternatively, improved survival after stroke, or an increased prevalence of subclinical vascular disease in the absence of overt stroke, might result in more individuals in the population with increased risk of dementia.

New medications and other therapies for cardiovascular disease introduced since the early 1990s (e.g., wider use of antihypertensive and statin medications) may have contributed to a reduction in myocardial infarction and stroke over the past 20 years $[12,13]$. Along with the likelihood of treatment, the prevalence of hypertension and diabetes has also increased. The prevalence of hypertension in African Americans aged $>65$ years increased from $73.0 \%$ to $83.0 \%$, and the prevalence of diabetes increased from $26.0 \%$ to $36.0 \%$ between years 1994 and 2002 [14,15]. The increased prevalence of hypertension, diabetes, and obesity may have contributed to an increased risk for cognitive decline and dementia, although the negative effect of diabetes and obesity on brain health may not become evident until future decades.

The increasing level of education among older adults over the past 20 years may have influenced the prevalence and outcomes of dementia. The proportion of adults aged $\geq 65$ years with a high school diploma increased from $53.0 \%$ in 1990 to $72.0 \%$ in 2003 , whereas the proportion with a college degree increased from $11.0 \%$ to $17.0 \%$ during this same period [16]. More years of formal education are associated with a reduced risk of dementia [17], likely through multiple causal pathways, including a direct effect on brain development and function (i.e., the building of "cognitive reserve"), better health behaviors, and the general health advantages of having more wealth and social opportunities [18].

The wealth of older adults has also increased significantly, with median household net worth for those aged $\geq 65$ years increasing from $\$ 119,000$ in 1989 to $\$ 196,000$ in 2005 (in constant 2005 dollars) [18]. Like education, more wealth is associated with lower levels of disability throughout the life course and may have contributed to declining levels of dementia over the past 20 years. In addition, there has been a widespread general increase in intelligence quotient (IQ) score, known as the Flynn effect. Mean IQ score in the United States was estimated to have increased 13.8 points in the 46 years from 1932 to 1978. Changes in environmental factors, education, and socioeconomic status have been offered as possible explanations; however, the precise mechanisms underlying the trend in IQ remain unknown [19].

In existing projections of future prevalence of $\mathrm{AD}$ in the U.S. population, the risk of $\mathrm{AD}$ is presumed to remain constant $[20,21]$. The increase in numbers is attributable to the continued increase in size of the older population, especially the oldest old (those aged $\geq 85$ years). The research reported in the present study examines whether there is also a trend in the underlying incidence or prevalence which could alter estimates of the number of subjects affected by $\mathrm{AD}$, dementia, or cognitive impairment in the future. Trends in prevalence may indicate changes in either incidence or survival. By contrast, trends in incidence would suggest changes in risk. Existing studies of trends in the incidence or prevalence of dementia and $\mathrm{AD}$ reported conflicting results. Rorsman et al found no statistically significant trend in the incidence of dementia and $\mathrm{AD}$ comparing the period between 1947 and 1957 with the period between 1957 and 1972 in a Swedish population study [22]. Similarly, there were no detectable trends in the incidence of dementia or AD comparing the period between 1975 and 1984 in Rochester, Minnesota [23]. By contrast, Manton et al found a decline in the prevalence of dementia in the United States using data from the National Long-Term Care Study between 1982 and 1999 [24]. Therefore, it remains unclear whether the incidence or prevalence of $\mathrm{AD}$, dementia, or cognitive impairment has increased, decreased, or remained stable over time in the United States. 
In this article, U.S. trends data are presented from three community-based studies and from a fourth study in a national sample. Rocca, Petersen, and Knopman report on trends in the incidence of dementia and AD for Rochester, Minnesota, for the period 1975 through 1994. Hebert and Evans report trends in the incidence of $\mathrm{AD}$ from the Chicago Health and Aging Project (CHAP) for the years 1997 through 2008. Hall, Gao, and Unverzagt compare prevalence figures for dementia and AD for 1992 versus 2001 in two samples of African Americans residing in Indianapolis, Indiana. Langa and Larson investigated trends in the prevalence of cognitive impairment for the data collection waves from 1993 and 2002 using national data from the Health and Retirement Study (HRS). In the Discussion section, White provides a synthesis of these findings and gives his perspective on this important topic.

\section{Secular trends in the incidence of dementia and $A D$ in Rochester, Minnesota: 1975 through 1994}

Incidence rates for dementia and $\mathrm{AD}$ from the population of Rochester, Minnesota, were previously reported for three different periods. Data were initially published for the 10 years 1975 through 1984 [23], then for the 5 years 1985 through 1989 [25], and most recently, for the 5 years 1990 through 1994 [26]. In this report, these data were combined over a total of 20 years (1975 through 1994) to investigate secular time trends in the incidence of dementia in a welldefined U.S. population.

The current study was made possible by the medical records-linkage system maintained by the Rochester Epidemiology Project (REP) [27]. Medical care for the populations of Rochester and Olmsted County, Minnesota, is provided largely by the Mayo Clinic at the primary, secondary, and tertiary levels. Additional healthcare providers in the community participate in the REP, which links essentially all medical information from the local population (the medical records-linkage system). Each provider in the community uses a dossier system (or unit record), in which all medical information for each individual is accumulated in a single record. Medical diagnoses and surgical interventions from the dossier are routinely abstracted and coded using the hospital version of the International Classification of Diseases, Adapted and other coding systems [27,28], and are entered into computerized indexes. Therefore, individuals with a given condition can be identified using diagnostic codes.

The indexes of the REP were searched for a list of specific diagnostic codes that might indicate dementia. This list expanded over time, from 112 codes for the 1975 through 1984 study [23] to 132 codes for the 1990 through 1994 study [26]. Any subject who received at least one dementia code during the study years was considered a potential case. All medical records of each potential case were screened by a specifically trained nurse abstractor and, when applicable, reviewed by a neurologist with special expertise in dementia. The neurologist confirmed the presence of dementia, classified the dementia by type, and determined the year of onset. Because cases of dementia in the general population may remain undetected for several years but may ultimately be diagnosed at some point during the natural history of the disease, all medical records of subjects who received one of the diagnostic codes of interest during a 6-year period following the last year of the study interval were also reviewed. The use of this extended capture time frame should have ensured a more complete case ascertainment $[23,25,26]$.

The diagnostic criteria for dementia were not entirely standardized because the REP data were collected as part of routine medical care rather than as part of a research project; however, the ad hoc criteria that were used are similar to those of the Diagnostic and Statistical Manual of Mental Disorders-III-Revised for dementia [29], and to those of the National Institutes of Health for AD [30]. Some, but not all, of the subjects affected by dementia underwent psychometric testing, imaging, or other research tests [23].

The total population living in Olmsted County, Minnesota, was approximately 110,000 at the 1990 U.S. Census (last census included in this study period), was evenly split between men and women (49.0\% men), and included approximately 11,000 subjects aged $\geq 65$ years. The population was primarily white of European descent. The analyses presented in this study refer to the incidence of dementia restricted to the city of Rochester rather than the full county [23].

The cases of dementia identified through the medical records-linkage system were used as numerators and U.S. Census data as denominators. However, the census data were corrected by removing prevalent cases of dementia to avoid miscounting the subjects at risk. More details about the calculation of incidence rates are provided elsewhere [23]. Time trends were investigated graphically using agespecific curves for 5-year age classes. Analyses were conducted for dementia and AD separately and included both genders combined because no consistent gender pattern was observed [23]. To increase the stability of the estimates, 3 -year moving average incidence rates were graphed. In addition, a series of birth cohort analyses were conducted using the methods described elsewhere [23]. The aims of these reanalyses were mainly descriptive. Because the study covered the target population completely, no sampling was involved, and statistical tests may not be appropriate to interpret the findings $[23,31,32]$. However, some statisticians argue that despite the descriptive aims of the study and the lack of sampling, statistical testing of the time trends can be performed.

Figure 1 shows the trend over 20 years for dementia in five age groups: 70 to 74,75 to 79,80 to 84,85 to 89 , and 90 to 94 years. For all the age groups, the incidence rates showed inconsistent fluctuations (ups and downs) over the 20 years of the study. However, there was some evidence for a declining trend in the second decade of the study, especially for the age groups 80 to 84,85 to 89 , and 90 to 94 


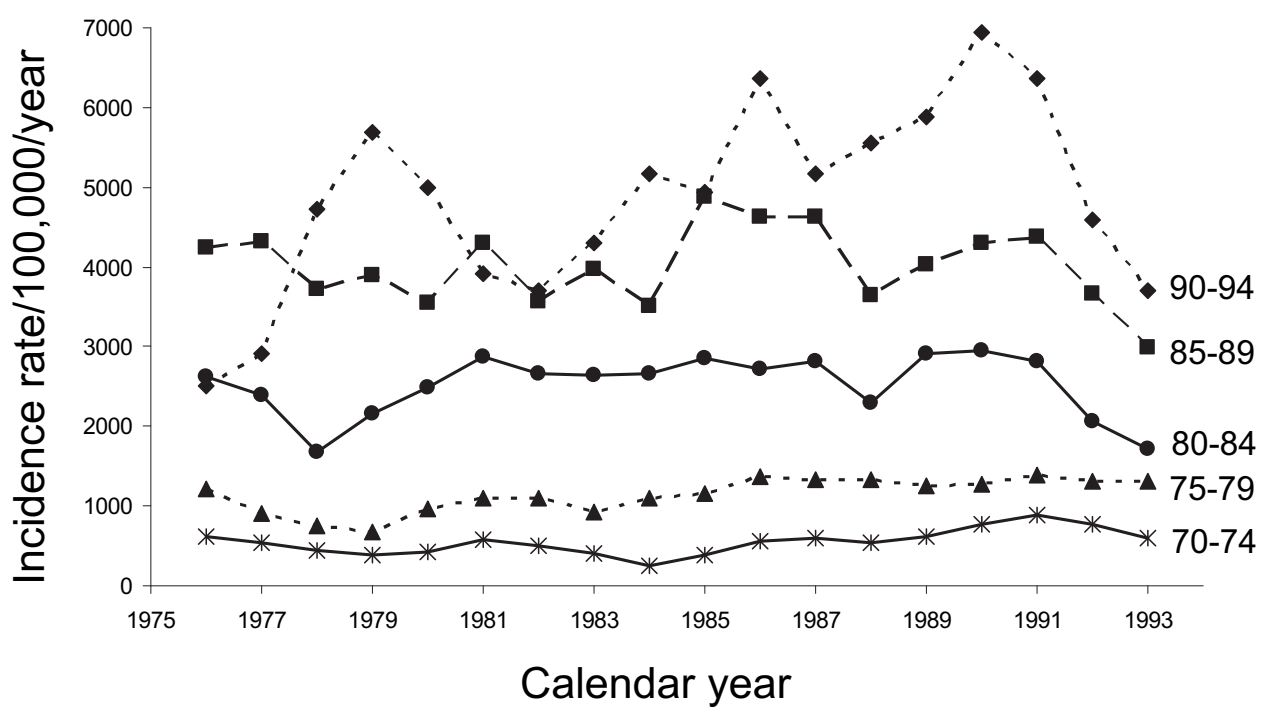

Fig. 1. Time trends in age-specific incidence rates of dementia in men and women combined from 1975 through 1994 (moving 3-year average incidence rates per 100,000 person-years): Rochester, Minnesota.

years. When Poisson regression was used to test for secular trends, there was no statistically significant calendar year effect for the entire 20-year period. However, there was a small but statistically significant decreasing trend in the second decade of the study, 1985 to 1994 (relative risk [RR], 0.97 per calendar year; 95\% confidence interval [CI], 0.95-0.99; $P=$ .004). This finding suggests a $3.0 \%$ decline in the incidence of dementia per year or a $30.0 \%$ decline over 10 years.

Figure 2 shows the corresponding time trends of $\mathrm{AD}$ for the same five age groups over the same 20-year time span. Not surprisingly, the trends for AD were similar to those for all types of dementia combined because $\mathrm{AD}$ is the most common type of dementia-about $74.0 \%$ to $75.0 \%$ of the cases [5]. Poisson regression analyses showed the same patterns of statistical significance as for dementia of all types. However, the numbers were too small to investigate the trends for vascular dementia (or for all of the non-AD types of dementia pooled).

Figure 3 shows the birth cohort curves for dementia (left panel) and $\mathrm{AD}$ (right panel). No obvious birth cohort effects were observed in this population. Different birth cohorts experienced the same incidence of dementia or AD when reaching the same age in different calendar years.

In conclusion, there were no secular trends in the incidence of dementia when considering the full study period and there were no differences in incidence across the 10 birth

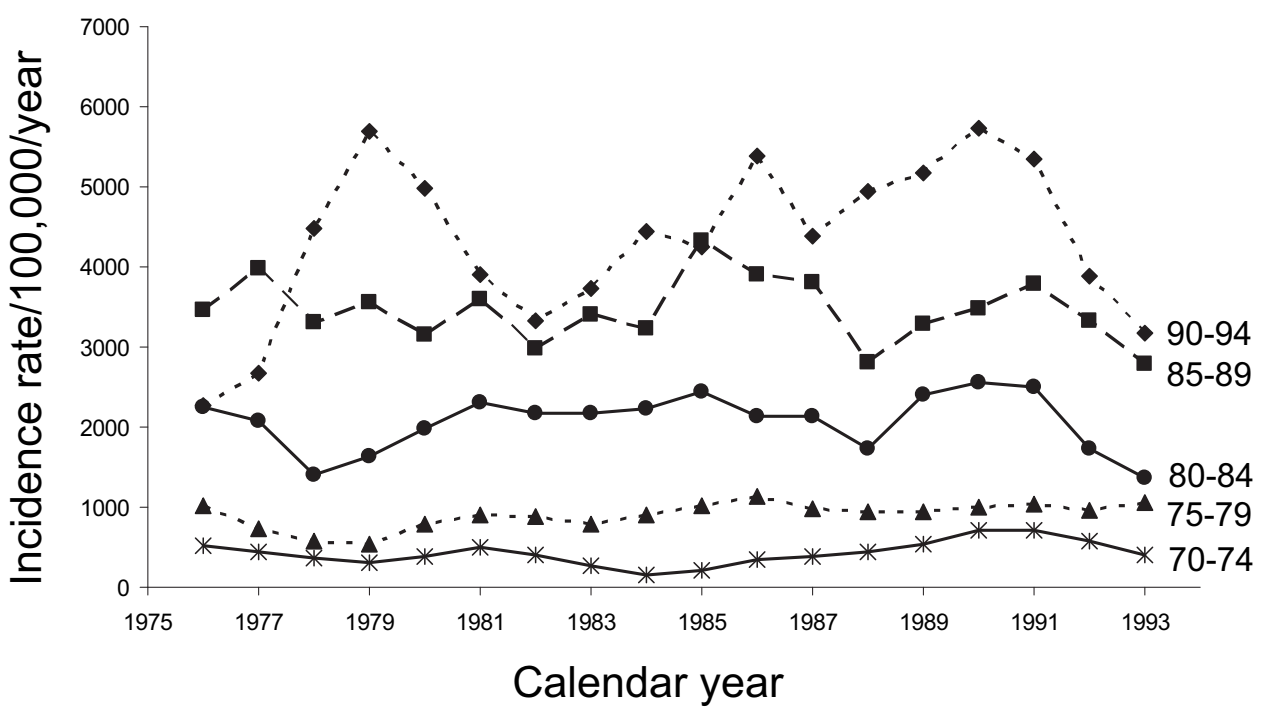

Fig. 2. Time trends in age-specific incidence rates of Alzheimer's disease (AD) in men and women combined from 1975 through 1994 (moving 3-year average incidence rates per 100,000 person-years): Rochester, Minnesota. 

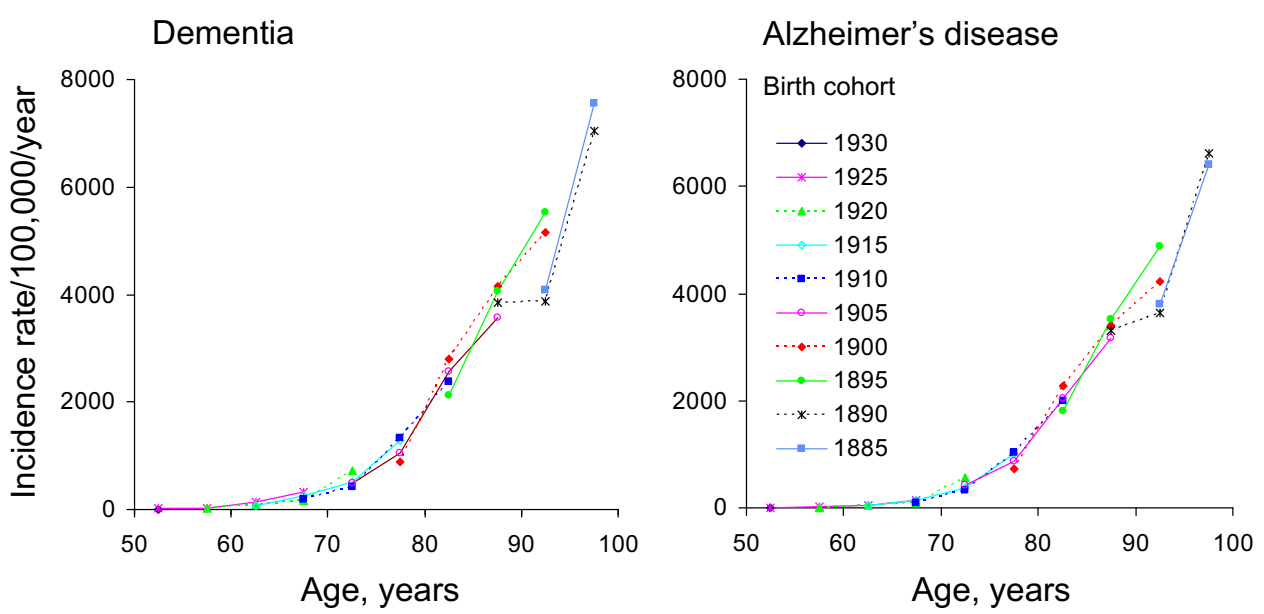

Fig. 3. Birth cohort analyses of age-specific incidence rates in men and women combined for dementia (left panel) and AD (right panel): Rochester, Minnesota. The central year of each birth cohort served as the cohort label. Ten 5-year birth cohorts from 1885 through 1930 were considered.

cohorts included in the study. However, the data may suggest a declining trend in the second decade of the study, between 1985 and 1994. This decline was evident for dementia overall as well as for AD. The interpretation of these trends remains uncertain.

First, these trends may simply be a chance finding and would not be confirmed if incidence rates for a longer time window were available. Second, the trends may be an artifact caused by some changes in the assignment of diagnostic codes for dementia over time, or in the linkage and storage of data in the REP. However, there were no changes in coding schemes for dementia in the REP between 1985 and 1994. In addition, there were no sizeable changes in patterns of referral to nursing homes inside or outside Olmsted County. Finally, the recognition of dementia by families and by practicing physicians was expected to increase rather than decrease during the period between 1985 and 1994 as a result of increased research activity, increased numbers of publications, and increased media attention focused on dementia.

Second, the declining trends may be genuine and may indicate some changes in risk factors for dementia over time. The major mechanisms of dementia can be grouped into two broad categories, that is, degenerative and vascular. It has been suggested that both degenerative and vascular mechanisms are involved in the pathogenesis of all cases of dementia and that their clinical separation is artificial [11]. Thus, each patient with dementia falls within a spectrum ranging from pure degenerative dementia to pure vascular dementia, and changes in the incidence of stroke may precede and determine changes in the incidence of dementia. Trends in the incidence of stroke may be responsible for the trends reported here [33]. The average incidence rates of stroke in Rochester, Minnesota, declined by $46.0 \%$ comparing the period between 1950 and 1954 with the period between 1975 and 1979. The incidence rates stabilized in the 1970s but increased by $17.0 \%$ in the period between 1980 and 1984 [34]. The incidence rates of stroke remained stable in the period between 1985 and 1989 [35]. Trends in the incidence of stroke after 1989 are not available in this community. The relationship between trends in stroke incidence and trends in dementia incidence needs further study. In addition, other trends in risk or protective factors such as education, income, or use of certain medications may also have played a role in the decline.

If the trends in the incidence of dementia between 1985 and 1994 are genuine, they have major implications for research $[7,33]$ and for public health [3]. Therefore, these findings need to be replicated in other populations and should be extended to more recent years (after 1994). The REP offers a unique opportunity to further explore these trends.

\section{Short-term trends in the incidence of $A D$ in the Chicago Health and Aging Project: 1997 to 2008}

Incidence trends were obtained using data from CHAP [36]. CHAP is a population-based study in Chicago with substantial numbers of both African American and white participants. The study began in 1993, when all residents aged $\geq 65$ years in a defined geographic area were invited to participate. Over time, additional people were invited to participate as they turned 65 years old. In this way, the number of living subjects remains fairly constant (between 6000 and 7000) and the full age distribution of the population is maintained. Data were collected in 3-year cycles. Any combination of two or more successive cycles was methodologically similar to a standard prospective cohort study (Fig. 4). Individuals were determined to be disease-free at the first of these cycles and a stratified random sample was evaluated for incidence of disease at the second of these cycles, 3 years later [37]. There have been a total of four cohort intervals. Evaluations for incident disease have been performed approximately continuously since the beginning of 


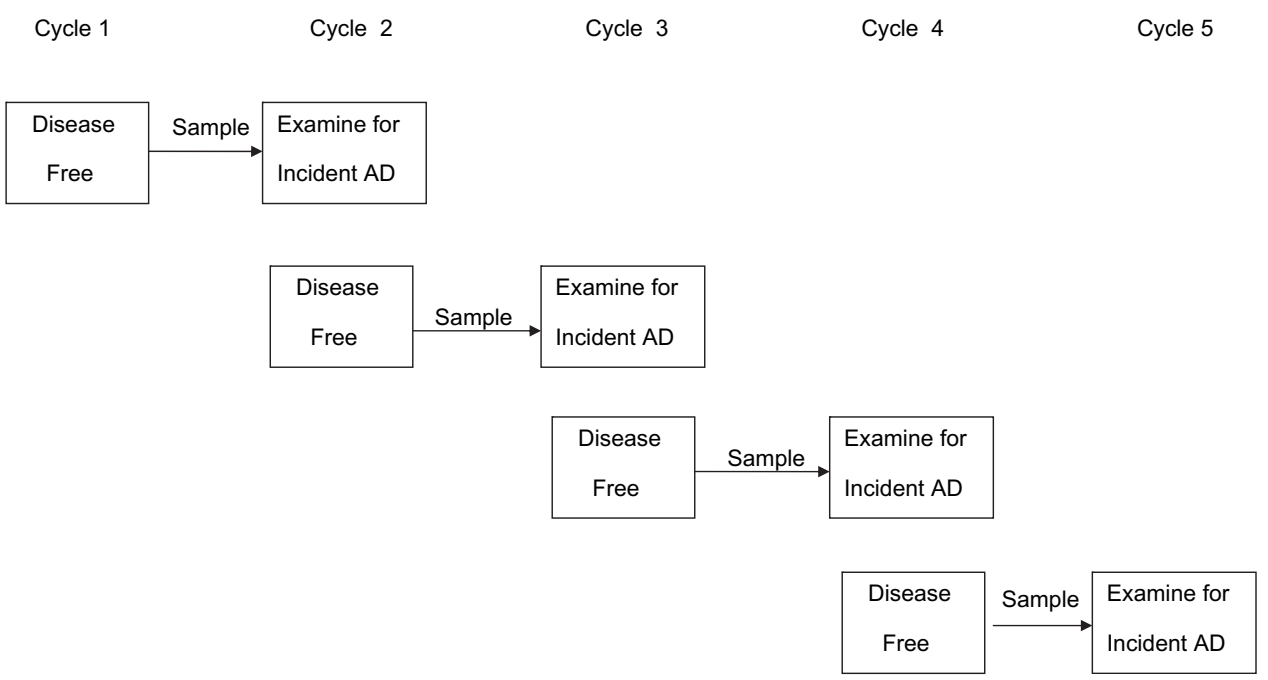

Fig. 4. Study design for determining incidence of AD in the Chicago Health and Aging Project.

the second cycle in 1997, for a total of 11 years. The total number of people who have contributed data to the project increased over time and is presently a little $>10,000$.

These analyses used data from subjects receiving clinical evaluation in the second (first incident) data collection cycle through the fifth cycle, between 1997 and 2008. A total of 1695 subjects were evaluated over this period and 360 developed incident AD. The mean age of the sample was 80.0 years (standard deviation [SD], 5.8). The mean education level was 12.9 years (SD, 3.5), which is similar to the community population and to the U.S. average. Most subjects were women $(61.6 \%)$ because women have a longer average survival. African Americans represented $52.5 \%$ of the sample.

The date of evaluation was used as a predictor in logistic regression analyses of the probability of incident $\mathrm{AD}$ to identify time trends. A logistic model rather than a survival model was used because the window for development of incident disease, that is the 3 years between data collection cycles, was fairly constant across subjects and without systematic variation over the course of the study. The model adjusted for age, gender, education, race, and interval from the visit when subjects were designated disease-free to the visit when the disease was first diagnosed. The analysis was weighted to reflect the full population from which the samples were drawn and used jackknife variance to correct for the stratified design. Table 1 shows the results of the analysis. If there is a trend toward increased or decreased diagnosis of $\mathrm{AD}$, the calendar year in which the diagnosis was made should appear as a significant variable. The result was null with an odds ratio (OR) of 0.97 and a $95 \%$ CI of 0.90 to $1.04(P=.41)$. Thus, there was no relation of calendar year of evaluation to disease incidence, suggesting no change in incidence over time.

This study had both strengths and limitations. The strengths were that the sample was population-based and biracial. The clinical evaluations for incidence of $\mathrm{AD}$ were structured and uniform throughout the study. The analytic methods made it possible to directly examine the change in disease risk over time. The weaknesses of this study limit the generalizability of the findings. The population was only one community and the time of observation was limited to 11 years. The data collection cycles themselves were approximately 3 years in duration, so cases with onset occurring over an atypically lengthy period or cases with death shortly after onset would be less likely to be identified. Perhaps the strongest limitation is that small, difficult-to-detect, changes in risk of $\mathrm{AD}$ over time could have a substantial effect if they were to continue over a long period. Even a large study over more than a decade such as the present one cannot convincingly exclude the possibility of such small changes.

\section{Prevalence of dementia and $\mathrm{AD}$ in African Americans in Indianapolis, Indiana: 1992 versus 2001}

The Indianapolis-Ibadan Dementia Project was designed to compare morbidity levels and potential risk factors for dementing disorders in population-based samples of African Americans in Indianapolis, Indiana, and Yoruba in Ibadan, Nigeria. This project was initiated in 1992 and provided

Table 1

Results of the logistic model predicting probability of incident Alzheimer's disease: Chicago Health and Aging Project [36]

\begin{tabular}{lllc}
\hline Predictor & OR & $95 \%$ CI & $P$ value \\
\hline Calendar year & 0.97 & $0.90-1.04$ & .41 \\
Age at evaluation (years) & 1.12 & $1.08-1.16$ & $<.001$ \\
Gender, men & 1.23 & $0.83-1.82$ & .29 \\
Race/ethnicity, African Americans & 1.77 & $1.19-2.63$ & .005 \\
Education (years) & 0.94 & $0.89-1.00$ & .07 \\
Interval for disease development* & 0.91 & $0.76-1.09$ & .29 \\
\hline
\end{tabular}

Abbreviations: OR, odds ratio; 95\% CI, 95\% confidence interval.

* Time in years from the visit when last found to be disease-free to the visit when first diagnosed with Alzheimer's disease. 
a unique opportunity to compare prevalence estimates over a decade in community-dwelling elderly African Americans. The population-based sample which was originally recruited in 1992 was enriched in 2001 to replace those participants who had died or were otherwise lost to followup. The aim in this section is to report a comparison of the prevalence of dementia and AD for the 1992 and 2001 cohorts, and also to report on differences in the demographic and medical profiles of the cohorts.

In 1992, interviewers went to randomly sampled addresses to enroll self-identified African Americans aged $\geq 65$ years; of 2582 eligible, 2212 enrolled $(9.6 \%$ refused, $4.7 \%$ were too sick) [38]. In 2001, Medicare lists were used for African Americans aged $\geq 70$ years; of 4260 eligible, 1892 (44.0\%) enrolled, 1999 (47.0\%) refused; the remainder were lost for other reasons. Informed consent was obtained from study participants before each interview. The study was approved by the Institutional Review Board at Indiana University School of Medicine.

The main study followed a two-stage design. In the first stage, all participants had an in-home interview. In the second stage, there was a full diagnostic clinical assessment in a subgroup of participants selected from the larger cohort on the basis of performance on the screening interview (good, intermediate, poor). Subjects were selected from all performance groups.

The screening interview relied on an instrument known as the Community Screening Interview for Dementia (CSID). It was specially developed for comparative epidemiological studies of age-associated dementias in different populations. The CSID had good sensitivity and specificity for discriminating dementia from no dementia in community-dwelling populations. Inter-rater reliability and 2-week test re-test reliability were also good [39]. The instrument included a standardized interview with a close relative about cognitive function and activities of daily living. The instrument also collected information about medical history, alcohol and tobacco use, and current medications (recorded by inspecting containers).

Clinical assessments were conducted in a home visit by a physician and psychometrician. The assessment included the following: (1) Consortium to Establish a Registry for Alzheimer's Disease neuropsychological test battery using normative values by education level, developed for elderly African Americans [40]; (2) standardized health examination and assessment of function; (3) semi-structured informant interview based on the Cambridge Examination for Mental Disorders of the Elderly adapted for American populations [41]; (4) request for medical records including neuroimaging. Diagnostic categories were dementia, cognitive impairment not dementia, or normal. Diagnosis was made in a consensus conference of clinicians reviewing all clinical data. Clinicians were kept blind regarding CSID scores. Standard diagnostic criteria were used [29,30,42,43]. Blood samples were collected in $10 \mathrm{~mL}$ (ethylenediaminetetraacetic acid) vacutainer tubes. The samples were centrifuged and red blood cells, buffy coat, and plasma were separated. Deoxyribonucleic acid was extracted from the buffy coat using standard protocols, and apolipoprotein E (APOE) genotype was obtained through Hhal digestion of amplified products [44]. A separate informed consent was obtained before blood collection.

T-tests and chi-square tests were used to compare the cohorts on continuous and categorical variables, respectively. They were also used to compare CSID poor performance group subjects with and without clinical assessment from each cohort. The age-specific prevalence estimates of dementia and $\mathrm{AD}$ were calculated using smoothing through logistic regression in which the probability of dementia or $\mathrm{AD}$ was modeled as a function of age, gender, and CSID performance group, and predicted probability from the model was used in the estimates [45]. Weighted logistic regression was used to account for different probabilities of selection into the sample for clinical assessment in different performance groups. Standard errors for the age-specific prevalence estimates were approximated using variance-covariance derived from the weighted logistic model. The $95 \%$ CIs for the prevalence estimates were constructed on the basis of asymptotic normality. The 1990 U.S. Census data for African Americans in Indianapolis were used for direct age standardization of the prevalence figures. The 1992 prevalence estimates were compared with the 2001 prevalence figures using weighted logistic models with an indicator variable for cohorts.

Table 2 shows the demographic characteristics, medical history, medication use, and APOE $\varepsilon 4$ status for the 1992 cohort compared with the 2001 cohort. Table 3 shows the age-

Table 2

Comparisons of demographics, medical history, and APOE genotype between the 1992 and 2001 cohorts: African American sample, Indianapolis, Indiana

\begin{tabular}{lccc}
\hline Variables & $\begin{array}{l}1992 \\
(\mathrm{n}=1500)\end{array}$ & $\begin{array}{l}2001 \\
(\mathrm{n}=1892)\end{array}$ & $\begin{array}{l}P \\
\text { value }\end{array}$ \\
\hline Mean age (SD) & $77.4(6.0)$ & $76.8(5.6)$ & .004 \\
Mean years of education (SD) & $9.3(3.2)$ & $11.3(2.7)$ & $<.0001$ \\
Female (\%) & 65.3 & 64.8 & .72 \\
Rural childhood residency (\%) & 34.2 & 24.8 & $<.0001$ \\
Ever used alcohol regularly (\%) & 30.6 & 36.1 & .001 \\
Ever smoked (\%) & 59.5 & 54.6 & .004 \\
Self or informant-reported history of & & & \\
Cancer (\%) & 11.9 & 16.7 & .0001 \\
Parkinson's disease (\%) & 1.1 & 1.0 & .61 \\
Heart attack (\%) & 15.6 & 14.0 & .20 \\
Hypertension (\%) & 64.7 & 75.2 & $<.0001$ \\
Diabetes (\%) & 24.4 & 29.3 & .002 \\
Stroke (\%) & 12.3 & 15.8 & .003 \\
Head injury (\%) & 8.7 & 8.9 & .84 \\
Depression (\%) & 6.9 & 11.1 & $<.0001$ \\
Medication usage & & & \\
Antihypertensive (\%) & 44.2 & 76.0 & $<.0001$ \\
Antidiabetic (\%) & 14.4 & 21.6 & $<.0001$ \\
Statins (\%) & 1.6 & 24.1 & $<.0001$ \\
Genetic testing* & & & .16 \\
APOE $\varepsilon 4$ allele carrier $(\%)$ & 39.9 & 36.2 & \\
\hline
\end{tabular}

* Genetic testing was available for 456 subjects in 1992 and 1133 subjects in 2001. 
Table 3

Age-specific prevalence of dementia and Alzheimer's disease, and overall standardized prevalence: African American sample, Indianapolis, Indiana

\begin{tabular}{|c|c|c|c|c|c|c|c|c|}
\hline \multirow[t]{3}{*}{ Age group (years) } & \multicolumn{4}{|l|}{1992} & \multicolumn{4}{|l|}{2001} \\
\hline & \multicolumn{2}{|l|}{ With dementia } & \multicolumn{2}{|l|}{ With AD } & \multicolumn{2}{|l|}{ With dementia } & \multicolumn{2}{|l|}{ With AD } \\
\hline & Prevalence $(\%)$ & $95 \% \mathrm{CI}$ & Prevalence $(\%)$ & $95 \% \mathrm{CI}$ & Prevalence $(\%)$ & $95 \% \mathrm{CI}$ & Prevalence $(\%)$ & $95 \% \mathrm{CI}$ \\
\hline $70-74$ & 2.5 & $1.6-3.4$ & 1.7 & $0.9-2.4$ & 1.9 & $0.9-2.9$ & 1.3 & $0.4-2.2$ \\
\hline $75-79$ & 4.9 & $3.5-6.2$ & 3.5 & $2.3-4.8$ & 4.7 & $2.2-7.2$ & 4.0 & $1.6-6.4$ \\
\hline $80-85$ & 9.7 & $7.3-12.1$ & 7.8 & $5.5-10.1$ & 10.3 & $3.6-16.9$ & 9.4 & $2.4-16.4$ \\
\hline $85+$ & 17.5 & $12.8-22.2$ & 15.8 & $11.0-20.6$ & 23.2 & $4.7-41.6$ & 22.5 & $4.6-40.4$ \\
\hline
\end{tabular}

Abbreviations: 95\% CI, 95\% confidence interval; AD, Alzheimer's disease.

* Direct standardization by age for subjects aged $\geq 70$ years using 1990 U.S. Census data for African Americans in Indianapolis, Indiana.

specific prevalence of dementia and possible or probable $\mathrm{AD}$ for each cohort. Results of the weighted logistic models comparing the prevalence of dementia and AD for the two cohorts showed no significant differences (dementia, $P=$ .35; AD, $P=.26$ ). For the 2001 cohort, comparisons were made for those who enrolled and those who did not, using information from the Medicare lists. Those who were not enrolled were significantly older (mean age, 79.1 years; SD, 7.4) compared with those who did enroll (mean age, 76.8 years; SD, 5.6; $P<.0001$ ). A higher proportion of women enrolled $(64.8 \%$ vs $59.6 \%, P=.0006)$. For the clinical assessment phase, the study participants in the CSID poor performance group who were not clinically assessed in 1992 (n $=88)$ and $2001(\mathrm{n}=47)$ did not differ significantly from those who were assessed, in regard to gender, age, and informant scores. The cognitive scores for those seen compared with those not seen were similar in 1992. However, the cognitive scores were slightly lower for the "not seen" group in $2001(P=.018)$.

There were no differences in the prevalence of dementia or AD for African Americans living in Indianapolis in 1992 versus 2001. The 2001 cohort had higher levels of hypertension, diabetes, and stroke, conditions associated with risk for dementia and $\mathrm{AD}$, but they also had higher levels of treatment. These differences are consistent with national trends for African Americans over this period [46]. The use of statins dramatically increased over this period (from $1.0 \%$ in 1992 to $24.0 \%$ in 2001). There were two conflicting trends with regard to cardiovascular risk factors; incidence rates went up but treatment levels also increased. The APOE $\varepsilon 4$ allele was associated with risk for $\mathrm{AD}$ in this population; it occurred in about the same frequency in the two cohorts and would not be expected to differentially affect the prevalence. Severity ratings of the diagnosed cases were compared, and no significant differences were found. There was an increase in the number of nursing homes in Indianapolis over the duration of the study; however, the percentage of African Americans residing in nursing homes remained constant at $6.0 \%$ at both periods $[47,48]$. Despite the higher frequency of risk factors and current treatments, no significant differences in prevalence between the two cohorts were found.
Limitations of the study include the change in recruitment strategies between 1992 and 2001, which resulted in higher refusal levels in 2001. In 1992, interviewers went door-todoor to randomly selected addresses. By contrast, the study area was greatly expanded to enrich the sample in 2001, and potential participants were initially contacted by mail and telephone, a much less personal approach. The effect of the increase in refusals on prevalence estimates is uncertain. Those who refused to participate were significantly older than those who enrolled, which could result in missing cases, thereby leading to an underestimation of prevalence. Although there was a higher proportion of women in the enrolled group, being a woman was not associated with higher risk [49]. Data on medical illnesses were based on self-report or informant report rather than by direct examination. The individuals who agreed to participate in $2001 \mathrm{had}$ slightly higher educational attainment than those of the 1992 cohort, and education may reduce the risk of dementia. This difference would possibly contribute to a lowering of the prevalence in 2001. If individuals with dementia were surviving longer in the community, this would lead to increased prevalence, whereas higher frequencies of institutionalization in nursing homes would lead to an underestimation. However, the percentages of African Americans living in nursing homes were the same in 1992 and 2001. Differential mortality for participants with dementia would lead to a decrease in prevalence estimates, but there is no evidence for changes in mortality risk in these cohorts [50].

In conclusion, there were no differences in the prevalence of dementia and AD between 1992 and 2001, despite significant demographic, medical history, and medical treatment differences in these two population-based cohorts of elderly African Americans.

\section{The prevalence of cognitive impairment among older adults in the Health and Retirement Study: 1993 versus 2002}

A large nationally representative study of older Americans was used to identify individuals with "cognitive impairment consistent with dementia" (CI-D). Data were obtained from the 1993 and 2002 waves of the HRS, a biennial, 
longitudinal, nationally representative survey of U.S. adults $[51,52]$. The main analytic goal was to identify two similar nationally representative cohorts of older individuals (aged, $\geq 70$ years) in 1993 and 2002, characterize their cognitive function using the same cognitive tests in each year, and then follow each cohort for 2 years to determine mortality for individuals with the following: (1) normal cognitive function; and (2) CI-D. The CI-D category was further sub-divided into mild CI-D and moderate/severe CI-D.

The HRS assesses cognitive function with a 35-point scale that includes an immediate and delayed 10-noun free recall test to measure memory; a serial seven subtraction test to measure working memory; a counting backward test to measure speed of mental processing; an object naming test to measure knowledge and language; and recall of the date, the president, and the vice-president to measure orientation. For self-respondents, the presence and severity of CI$\mathrm{D}$ were defined using this 35 -point cognitive scale. For those represented by a proxy (about $10.0 \%$ in each cohort), the proxy's assessment of the memory and judgment of the respondent were used to categorize the level of cognitive function.

The definitions and cut-points for these categories were based on previous studies with the HRS data [53], as well as the methods used for the Aging, Demographics, and Memory Study, which is a supplemental study of dementia in the HRS [54]. The validity of these categories is supported by the clear trends in functional limitations with which they are associated; the mean number of limitations in instrumental activities of daily living (preparing meals, grocery shopping, making phone calls, taking medications, managing money) in the normal, mild CI-D, and moderate or severe CI-D categories had an average of $0.4,1.1$, and 2.5 instrumental activities of daily living limitations, respectively $(P$ $<.001$ ). More detail on the HRS self-report and proxy cognitive measures is available at the HRS Web site [55].

The following sociodemographic measures were included in the analyses as independent variables: age (7079 years, 80-89 years, $\geq 90$ years), race (white, African American, Other), gender, education ( $<12$ years, 12 years, $13-15$ years, and $\geq 16$ years), potential caregiver network (a spouse and/or living children), and net worth (tertiles; expressed in 1993 dollars). The self-reported chronic medical conditions that were considered included stroke, diabetes, heart disease, hypertension, lung disease, cancer, psychiatric problems, smoking status, and obesity (self-reported height and weight resulting in a body mass index $\geq 30$ ).

Data from 1993 and 2002 were pooled and logistic regression models with a dichotomous dependent variable indicating whether an individual had CI-D (mild, moderate/ severe) were estimated. A linear trend variable that took the value of zero in 1993 and one in 2002 was included in the logistic regression models. An OR $<1$ for this trend variable would indicate a decrease in the prevalence of CI-D between 1993 and 2002. Seven separate logistic regression models with different sets of independent variables (e.g., demographic variables, education, cardiovascular risks, and other chronic conditions) were estimated to determine which variables were most significantly associated with change in the prevalence of CI-D between 1993 and 2002. All results were adjusted for the complex sampling design of the HRS using appropriate population weights [52]. Statistical analyses were performed with STATA (Release 8.0; Stata Corp, College Station, TX) and SUDAAN (Release 9.0; Research Triangle Institute, Research Triangle Park, NC).

Table 4 shows the characteristics of the 1993 and 2002 study cohorts. As compared with the 1993 cohort, the 2002 cohort was slightly older (mean of 77.8 vs 77.5 years), had significantly more years of education, and had higher net worth (in constant 1993 dollars). Individuals with less than a high school diploma (12 years of education) comprised $42.0 \%$ of the sample in 1993 , but only $31.0 \%$ in 2002 . On average, individuals in the 2002 cohort had almost 1 more year of education compared with those in the 1993 cohort (mean of 11.8 vs 11.0 years).

In the unadjusted analysis, there was a significant decrease in the prevalence of CI-D between 1993 and 2002, from $12.2 \%$ in 1993 to $8.7 \%$ in $2002(P<.001)$. Table 5 reports the results of seven different logistic regression models with the presence of CI-D (mild, moderate/severe) as the outcome variable, using pooled 1993 and 2002 data. The trend variable in the first row of the table represents the odds of CI-D in 2002 compared with 1993. Model 1 shows the statistically significant decline $(\mathrm{OR}, 0.68)$ in unadjusted CI-D prevalence noted earlier. When adjusting for age and gender differences between the two cohorts (Model 2), the trend toward decreased CI-D prevalence was slightly larger (the trend OR drops from 0.68 to 0.65 ) because of the older age of the 2002 cohort and the strong association of older age with increased odds of CI-D. Higher levels of education (Model 3) and net worth (Model 4) were associated with significantly lower odds of CI-D, and accounted for about $43.0 \%$ ( 15.0 percentage points) of the decrease in CI-D prevalence between 1993 and 2002 (i.e., the trend OR increased from 0.65 to 0.80 after adding education and net worth to the model).

In a large nationally representative survey of older Americans, the prevalence of CI-D decreased from $12.2 \%$ to $8.7 \%$ between 1993 and 2002, representing an absolute decrease of 3.5 percentage points, and a relative decrease of nearly $30.0 \%$. The decline in the prevalence of CI-D suggests that, overall, the combined effect of recent trends in medical, lifestyle, demographic, and social factors has been positive for the cognitive health of older Americans. Although the prevalence of some cardiovascular risk factors that are also associated with a higher risk of dementia increased significantly, other factors showed trends that favored a reduced prevalence of CI-D. Most importantly, individuals aged $\geq 70$ years in 2002 had significantly higher levels of education, on average, than those aged $\geq 70$ years in 1993 . The trend analyses suggest that increasing levels of education 
Table 4

Characteristics of the 1993 and 2002 study cohorts from the Health and Retirement Study

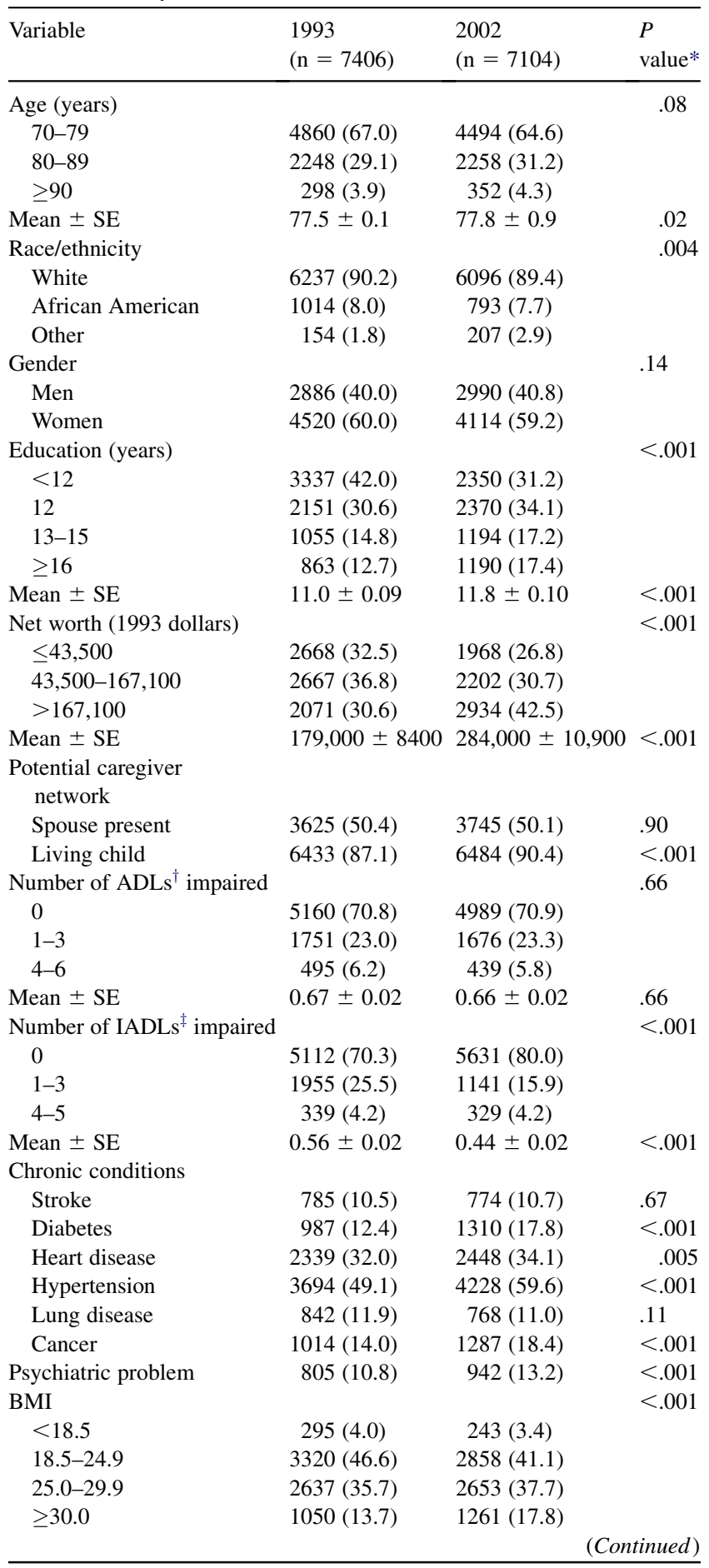

and net worth among older Americans explained about $40.0 \%$ of the observed relative decrease in CI-D prevalence between 1993 and 2002.

The relationship of temporal changes in various aspects of vascular risk to trends in CI-D is complex and will be important to track in the coming decades. Better control of hy-
Table 4

Characteristics of the 1993 and 2002 study cohorts from the Health and Retirement Study (Continued)

\begin{tabular}{lccc}
\hline Variable & $\begin{array}{l}1993 \\
(\mathrm{n}=7406)\end{array}$ & $\begin{array}{l}2002 \\
(\mathrm{n}=7104)\end{array}$ & $\begin{array}{l}P \\
\text { value* }\end{array}$ \\
\hline $\begin{array}{l}\text { Smoking status } \\
\quad \text { Never }\end{array}$ & $3508(47.8)$ & $3128(44.6)$ & $<.001$ \\
$\quad$ Former & $3113(42.5)$ & $3381(47.6)$ & \\
$\quad$ Current & $729(9.7)$ & $541(7.7)$ & \\
Respondent type & & & .73 \\
$\quad$ Self & $6621(89.7)$ & $6295(89.9)$ & \\
$\quad$ Proxy & $785(10.3)$ & $809(10.1)$ & \\
\hline
\end{tabular}

Abbreviations: SE, standard error; ADL, activities of daily living; IADL, instrumental activities of daily living; BMI, body mass index.

Values in parentheses are weighted percentages derived using the HRS respondent population weights to adjust for the complex sampling design of the HRS.

* $P$ value for $\chi^{2}$ or $t$-test for a significant difference in proportion or mean between years.

${ }^{\dagger}$ ADLs indicate Activities of Daily Living (eating, transferring, toileting, dressing, bathing, and walking across a room).

${ }^{\ddagger}$ IADLs indicate Instrumental Activities of Daily Living (preparing meals, grocery shopping, making phone calls, taking medications, managing money).

pertension and smoking, along with more effective drugs to control lipid risk, would be expected to delay onset of CI-D, whereas increasing prevalence of diabetes and obesity would be expected to have an opposite effect. In addition, the effects of the increase in diabetes prevalence between 1993 and 2002 on CI-D may take more time to become apparent in population studies like the HRS. This is an important area for future research, given the growing obesity epidemic and the associated increase in diabetes.

One important threat to the validity of this study is the nonrandom attrition from the HRS cohort. Specifically, if those with cognitive impairment are more likely to drop out of the study than those with normal cognitive function, the prevalence of cognitive impairment may decline over time as an artifact. To further examine the potential effect of differential loss to follow-up on the results, the baseline cognitive status of those who were lost to follow-up between the 1993 and 2002 waves of the study was examined (i.e., those who were not known to be dead and who did not provide either a selfinterview or proxy interview in 2002). Of the 7406 individuals included in the baseline 1993 survey, 453 (6.1\%) were lost to follow-up in 2002. Among this group, $6.3 \%$ of those who had normal cognitive function in 1993, $4.6 \%$ of those with mild CI-D in 1993, and $3.4 \%$ of those with moderate or severe CI-D were lost to follow-up. These results suggested that differential loss to follow-up was not an important reason for the lower prevalence of CI-D found in 2002, because the overall loss to follow-up rate was low (93.9\% of individuals were accounted for), and those with poorer cognitive function at baseline were actually somewhat less likely to be lost to follow-up by the 2002 wave.

The growth in the elderly population in the coming decades increases the public health importance of better 
Table 5

Odds ratios for presence of cognitive impairment in 1993 and $2002(\mathrm{n}=14,476)$ : Health and Retirement Study*

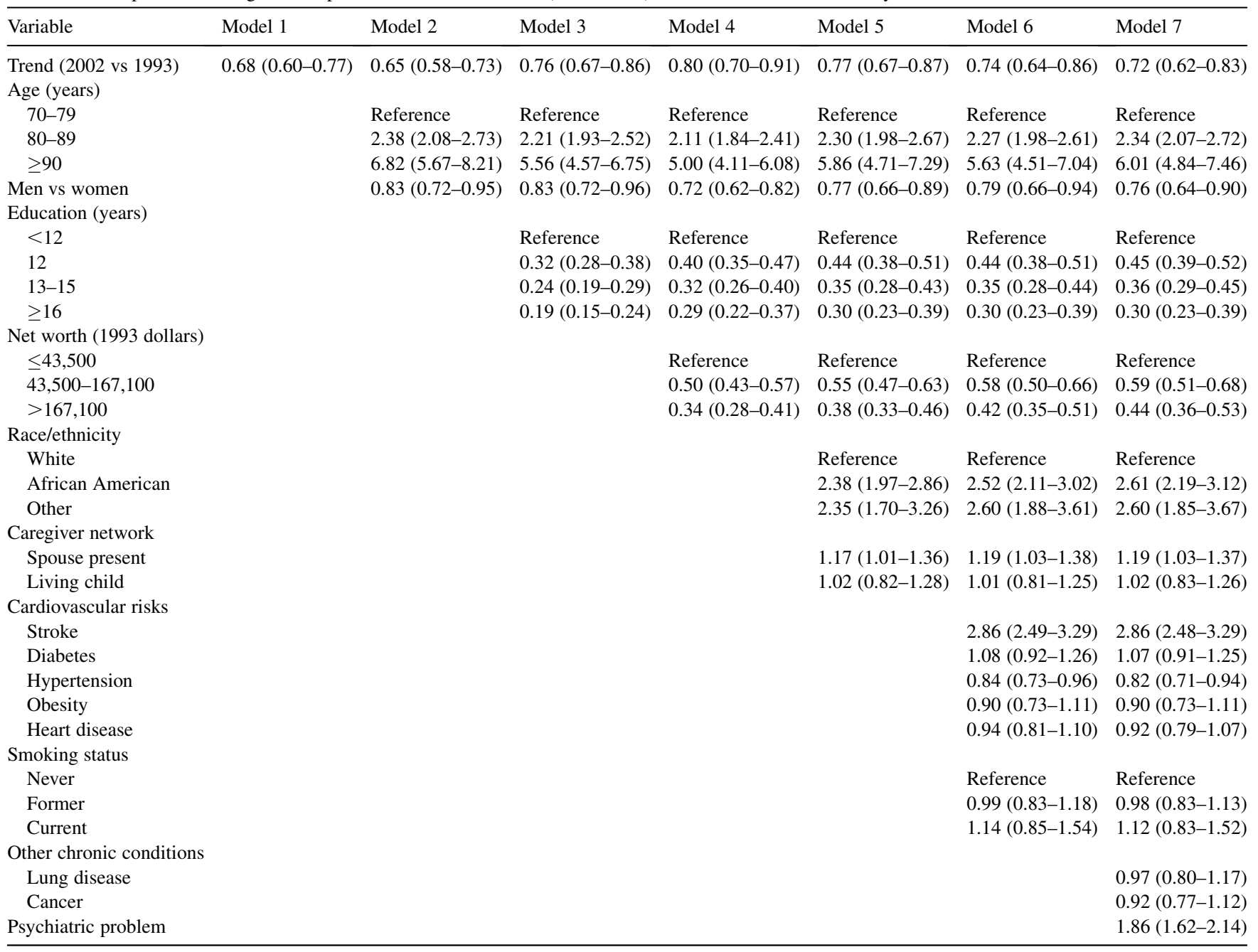

*Adjusted odds ratios derived using a logistic regression model with pooled $1993(\mathrm{n}=7393)$ and $2002(\mathrm{n}=7083)$ data with cognitive impairment (mild, moderate/severe) as the dependent variable. Values $>1$ indicate increased odds of cognitive impairment. $95 \%$ confidence intervals are in parentheses.

understanding trends in cognitive health and whether a societal investment in building and maintaining cognitive reserve through formal education in childhood, as well as continued cognitive stimulation during work and leisure in adulthood, may help limit the future burden of dementia, especially among the oldest old. A more detailed account of the work discussed in this section was published earlier [56].

\section{Discussion}

The four studies reported here represent complementary efforts to detect recent trends in the prevalence and incidence of $\mathrm{AD}$, dementia, and cognitive impairment. The impetus for these analyses includes their possible utility to understand determinants and risk factors for these endpoints and to better anticipate the quantity and type of social and healthcare needs of America's older citizens in the coming years. In particular, these analyses explored the notion that recent im- provements in cardiovascular and cerebrovascular disease risks might be reflected in changes in dementia rates.

Heart disease and stroke mortality rates have declined dramatically over the past 4 to 5 decades. By contrast, prevalence levels of obesity, diabetes, and hypertension have increased in recent years in the general population, from childhood through adult life. Mortality improvements are generally attributed to reductions in large artery atherosclerosis associated with reductions in cigarette smoking, better control of serum lipid levels, more effective recognition and treatment of diabetes and hypertension, and more aggressive public health approaches to nutritional and other lifestyle factors. The widespread use of aspirin or other medications has undoubtedly facilitated these trends as well. Depending on the balance between these divergent influences, the result could be either a reduction or an increase in dementia prevalence and incidence rates.

During the past 5 years, there has been a growing recognition that many cases of dementia in late life, including 
those that receive a clinical diagnosis of $\mathrm{AD}$, involve a combination of two or more independent pathological processes [57]. The most common combinations involve the Alzheimer process in concert with ischemic cerebrovascular disease and/or cortical Lewy bodies [58]. Autopsy studies have demonstrated that when the defining lesions of $\geq 2$ of these independent processes occur together, the probability that the person had been impaired or demented is additive (i.e., the overall probability reflects the added probabilities of each lesion alone) [59]. Dementing illnesses attributable to mixed pathologies become progressively more common with advancing age, as would be expected on the basis of the strong associations of all three of these processes with aging. A recent report that $17 \%$ of dementia cases in the Honolulu-Asia Aging Study were attributable to untreated or ineffectively treated midlife hypertension [60] supports the idea that improvements in rates of heart disease and stroke and their risk factors will lead to reduced rates of dementia. A demonstrable trend toward lower dementia rates in recent years would provide considerable support for the idea that public health interventions directed at reducing hypertension, smoking, and other risk factors for stroke and heart disease, and at improving general health, may yield the supplementary benefit of preventing some cases of cognitive impairment and dementia.

Three of the four reports presented here were from established community-based epidemiological studies of dementia, and all used appropriate clinical and research diagnostic criteria for dementia and AD. The Mayo Clinic report used data from the Rochester, Minnesota, population, analyzing rates for dementia diagnoses over a period of 20 years. CHAP used approximately 10 years of data for samples of mixed ethnicity from a defined community within Chicago, Illinois. The third report examined data from two cycles of a project examining rates of dementia in African American residents of Indianapolis, Indiana, over an interval of about 10 years. A major strength of all three studies is their excellent epidemiological designs, the expertise of the research teams, and the sophistication of analyses applied to their collected data. An inescapable weakness is that the time interval across which rates were compared is relatively short. If dementia rates, like heart and stroke mortality rates, have been gradually decreasing over the past 30 to 40 years, 10 or even 20 years may not provide enough time for that change to be statistically recognizable. Further, during the periods examined there have been changes in many social and cultural factors likely to influence endpoint assessments, including a decreasing enthusiasm for study participation, and the medical or general acceptability of a diagnosis of cognitive impairment. Although the Mayo Clinic investigators described a "blip" of lower rates of dementia in the second period (1985-1994), none of the three reports provided statistically significant evidence of a clear trend in rates of dementia or AD.

The fourth report presents an analysis of data from two waves of a nationally representative survey, the HRS. An al- gorithmic endpoint based on neuropsychological test scores was used to define cognitive impairment, rather than a criterion-based clinical or research diagnosis of dementia. A significant decline in the prevalence of cognitive impairment was observed between two waves of data collection separated by an interval of about 10 years.

Results from the HRS analysis provide quite strong support for decreasing rates of cognitive impairment among the elderly in the United States. Conversely, the findings reported from the other three studies do not support a definite change in recent decades. Given the current understanding of the likely importance of ischemic cerebrovascular disease and allied risk factors contributing to dementia and agingrelated cognitive decline, it seems reasonable to remain cautiously optimistic while continuing to work toward a full understanding of all factors determining the occurrence of dementia.

\section{Acknowledgments}

This combined effort was supported by National Insti tute on Aging grants R01 AG034676, U01 AG06786, R01AG011101, R03AG029652, R01AG009956, U01AG009740, R01AG015911，R01AG019805，R01AG027010，K08AG019180, U01AG006781, U01AG017155, U01AG019349. Also supporting the project were the Robert $\mathrm{H}$. and Clarice Smith and Abigail Van Buren Alzheimer's Disease Research Program at the Mayo Clinic, Rochester, Minnesota, and the Interfaculty Program for Health Systems Improvement at Harvard University, Cambridge, Massachusetts.

Rocca, Petersen, and Knopman acknowledge the contribution to the Mayo analyses by Ruth H. Cha, MS, and Brandon R. Grossardt, MS.

Hall, Gao, and Unverzagt thank the elderly participants of Indianapolis, Indiana, for their continued involvement with the Indianapolis-Ibadan Dementia Project. They also thank Millicent Pettaway for nursing supervision and data collection, and Jenna York for manuscript preparation.

Disclosure statement for authors: The authors have no conflicts to disclose. The sponsors had neither a role in the analysis or interpretation of these data, nor in the content of the paper. Appropriate approval procedures were used concerning human subjects.

\section{References}

[1] Kinsella K, Wan H. U.S. Census bureau: international population reports, P95/09-1, An Aging World: 2008. Washington, DC: U.S. Government Printing Office; 2009.

[2] Wimo A, Jonsson L, Winblad B. An estimate of the worldwide prevalence and direct costs of dementia in 2003. Dement Geriatr Cogn Disord 2006;21:175-81.

[3] Brookmeyer R, Johnson E, Ziegler-Graham K, Arrighi HM. Forecasting the global burden of Alzheimer's disease. Alzheimers Dement 2007;3:186-91.

[4] Rocca WA. Dementia, Parkinson's disease, and stroke in Europe: a commentary. Neurology 2000;54:S38-40. 
[5] Fratiglioni L, Rocca WA. Epidemiology of dementia. In: Boller F, Cappa S, eds. Handbook of Neuropsychology. 2nd ed, Vol. 6. Amsterdam, the Netherlands: Elsevier; 2001, p. 193-215.

[6] Corrada MM, Brookmeyer R, Paganini-Hill A, Berlau D, Kawas CH. Dementia incidence continues to increase with age in the oldest old: the 90+ study. Ann Neurol 2010;67:114-21.

[7] Querfurth HW, LaFerla FM. Alzheimer's disease. N Engl J Med 2010; 362:329-44.

[8] Fratiglioni L, Paillard-Borg S, Winblad B. An active and socially integrated lifestyle in late life might protect against dementia. Lancet Neurol 2004;3:343-53.

[9] Jedrziewski MK, Lee VM, Trojanowski JQ. Lowering the risk of Alzheimer's disease: evidence-based practices emerge from new research. Alzheimers Dement 2005;1:152-60.

[10] Grodstein F. Cardiovascular risk factors and cognitive function. Alzheimers Dement 2007;3:S16-22.

[11] Viswanathan A, Rocca WA, Tzourio C. Vascular risk factors and dementia: how to move forward? Neurology 2009;72:368-74.

[12] Mortality and life expectancy - mortality by underlying and multiple cause, ages 18+: US, 1981-2007 (Source: NVSS). Available at: http://www.cdc.gov/nchs/hdi.htm.

[13] Cutler DM, McClellan M. Is technological change in medicine worth it? Health Aff (Millwood) 2001;20:11-29.

[14] Health conditions-diabetes, ages 20+, US, 1988-2006 (Source: NHANES). Available at: http://www.cdc.gov/nchs/hdi.htm.

[15] Risk factors and disease prevention-hypertension, ages 20+: US, 1988-2008 (Source: NHANES). Available at: http://www.cdc.gov/ nchs/hdi.htm.

[16] Older Americans: key indicators of well-being. Available at: http:// www.agingstats.gov.

[17] Stern Y. Cognitive reserve and Alzheimer disease. Alzheimer Dis Assoc Disord 2006;20:S69-74.

[18] Cagney KA, Lauderdale DS. Education, wealth, and cognitive function in later life. J Gerontol B Psychol Sci Soc Sci 2002;57:P163-72.

[19] Flynn JR. The mean IQ of Americans: massive gains 1932 to 1978. Psychol Bull 1984;101:171-91.

[20] Brookmeyer R, Gray S, Kawas C. Projections of Alzheimer's disease in the United States and the public health impact of delaying disease onset. Am J Public Health 1998;88:1337-42.

[21] Hebert LE, Scherr PA, Bienias JL, Bennett DA, Evans DA. Alzheimer disease in the US population: prevalence estimates using the 2000 census. Arch Neurol 2003;60:1119-22.

[22] Rorsman B, Hagnell O, Lanke J. Prevalence and incidence of senile and multi-infarct dementia in the Lundby Study: a comparison between the time periods 1947-1957 and 1957-1972. Neuropsychobiology 1986;15:122-9.

[23] Rocca WA, Cha RH, Waring SC, Kokmen E. Incidence of dementia and Alzheimer's disease: a reanalysis of data from Rochester, Minnesota, 1975-1984. Am J Epidemiol 1998;148:51-62.

[24] Manton KC, Gu XL, Ukraintseva SV. Declining prevalence of dementia in the U.S. elderly population. Adv Gerontol 2005;16:30-7.

[25] Edland SD, Rocca WA, Petersen RC, Cha RH, Kokmen E. Dementia and Alzheimer disease incidence rates do not vary by sex in Rochester, Minnesota. Arch Neurol 2002;59:1589-93.

[26] Knopman DS, Petersen RC, Cha RH, Edland SD, Rocca WA. Incidence and causes of nondegenerative nonvascular dementia: a population-based study. Arch Neurol 2006;63:218-21.

[27] Melton LJ 3rd. History of the Rochester Epidemiology Project. Mayo Clin Proc 1996;71:266-74.

[28] Commission on Professional and Hospital Activities, National Center for Health Statistics. H-ICDA, Hospital Adaptation of ICDA. 2nd ed. Ann Arbor, MI: Commission on Professional and Hospital Activities; 1973.

[29] American Psychiatric Association, American Psychiatric Association Work Group to Revise DSM-III. Diagnostic and Statistical Manual of Mental Disorders: DSM-III-R. 3rd ed. Washington, DC: American Psychiatric Association; 1987.
[30] McKhann G, Drachman D, Folstein M, Katzman R, Price D, Stadlan EM. Clinical diagnosis of Alzheimer's disease: report of the NINCDS-ADRDA Work Group under the auspices of Department of Health and Human Services Task Force on Alzheimer's Disease. Neurology 1984;34:939-44.

[31] Anderson DW, Mantel N. On epidemiologic surveys. Am J Epidemiol 1983;118:613-9.

[32] Deming WE. Boundaries of statistical inference. In: Smith $\mathrm{H}$, Johnson NL, eds. New Developments in Survey Sampling. New York, NY: Wiley-Interscience; 1969:652-70.

[33] Gorelick PB, Erkinjuntti T, Hofman A, Rocca WA, Skoog I, Winblad B. Prevention of vascular dementia. Alzheimer Dis Assoc Disord 1999;13:S131-9.

[34] Broderick JP, Phillips SJ, Whisnant JP, O'Fallon WM, Bergstralh EJ. Incidence rates of stroke in the eighties: the end of the decline in stroke? Stroke 1989;20:577-82.

[35] Brown RD Jr, Whisnant JP, Sicks JD, O'Fallon WM, Wiebers DO. Stroke incidence, prevalence, and survival: secular trends in Rochester, Minnesota, through 1989. Stroke 1996;27:373-80.

[36] Hebert LE, Bienias JL, Aggarwal NT, Wilson RS, Bennett DA, Shah RC, Evans DA. Change in risk of Alzheimer disease over time. Neurology 2010;75:786-91.

[37] Bienias JL, Beckett LA, Bennett DA, Wilson RS, Evans DA. Design of the Chicago Health and Aging Project (CHAP). J Alzheimers Dis 2003;5:349-55.

[38] Hendrie HC, Osuntokun BO, Hall KS, Ogunniyi AO, Hui SL, Unverzagt FW, et al. Prevalence of Alzheimer's disease and dementia in two communities: Nigerian Africans and African Americans. Am J Psychiatry 1995; 152:1485-92.

[39] Hall KS, Ogunniyi AO, Hendrie HC, Osuntokun BO, Hui SL, Musick BS, et al. A cross-cultural community based study of dementias: methods and performance of the survey instrument, Indianapolis, U.S.A., and Ibadan, Nigeria. Int J Methods Psychiatr Res 1996;6:129-42.

[40] Unverzagt FW, Hall KS, Torke AM, Rediger JD, Mercado N, Gureje O, Osuntokun BO, Hendrie HC. Effects of age, education, and gender on CERAD neuropsychological test performance in an African American sample. Clin Neuropsychol 1996;10:180-90.

[41] Hendrie HC, Hall KS, Brittain HM, Austrom MG, Farlow M, Parker J, et al. The CAMDEX: a standardized instrument for the diagnosis of mental disorder in the elderly: a replication with a US sample. J Am Geriatr Soc 1988;36:402-8.

[42] World Health Organization. International Statistical Classification of Diseases and Related Health Problems. 10th Rev ed. Geneva, Switzerland: World Health Organization; 1992.

[43] What is the CDR? Available at: http://alzheimer.wustl.edu/cdr/ AboutCDR/aboutcdr.htm.

[44] Hixson JE, Vernier DT. Restriction isotyping of human apolipoprotein E by gene amplification and cleavage with HhaI. J Lipid Res 1990; 31:545-8.

[45] Beckett LA, Scherr PA, Evans DA. Population prevalence estimates from complex samples. J Clin Epidemiol 1992;45:393-402.

[46] Health care use and expenditures-personal health care expenditures of Medicare beneficiaries by chronic conditions and type of service, ages 65+: US, 1992-2005 (Source: MCBS). Available at: http:// www.cdc.gov/nchs/hdi.htm.

[47] Marion County Health Department II. Annual Reports by Nursing Homes; 1989.

[48] Census 2000 Summary File 1 (SF 1) 100-Percent Data, Detailed Tables, Marion County, Indiana. Sex by age (Black or African-American Alone) and Group Quarters Population by Age by Group Quarters Type (Black or African-American Alone). Available at: http:// factfinder.census.gov/servlet/DatasetMainPageServlet?_program = DEC\&_submenuId $=$ \&_lang $=$ en\&_ts $=$.

[49] Hall K, Gureje O, Gao S, Ogunniyi A, Hui SL, Baiyewu O, et al. Risk factors and Alzheimer's disease: a comparative study of two communities. Aust N Z J Psychiatry 1998;32:698-706. 
[50] Lane KA, Gao S, Hui SL, Murrell JR, Hall KS, Hendrie HC. Apolipoprotein $\mathrm{E}$ and mortality in African-Americans and Yoruba. J Alzheimers Dis 2003;5:383-90.

[51] Juster FT, Suzman R. An overview of the Health and Retirement Study. J Hum Resour 1995;30:S7-S56.

[52] Health and Retirement Study: a longitudinal study of health, retirement, and aging. Available at: http://hrsonline.isr.umich.edu/.

[53] Langa KM, Chernew ME, Kabeto MU, Herzog AR, Ofstedal MB, Willis RJ, et al. National estimates of the quantity and cost of informal caregiving for the elderly with dementia. J Gen Intern Med 2001; 16:770-8.

[54] Langa KM, Plassman BL, Wallace RB, Herzog AR, Heeringa SG, Ofstedal MB, et al. The Aging, Demographics, and Memory Study: study design and methods. Neuroepidemiology 2005;25:181-91.

[55] Wallace RB, Herzog AR, Weir DR, Ofstedal MB, Langa KM, Fisher GG, et al. Documentation of Cognitive Functioning Measures in the Health and Retirement Study. Ann Arbor, MI: Survey Research Center, University of Michigan; 2005. Available at: http://hrsonline.isr.umich.edu/ sitedocs/userg/dr-006.pdf.
[56] Langa KM, Larson EB, Karlawish JH, Cutler DM, Kabeto MU, Kim SY, et al. Trends in the prevalence and mortality of cognitive impairment in the United States: is there evidence of a compression of cognitive morbidity? Alzheimers Dement 2008;4:134-44.

[57] Schneider JA, Aggarwal NT, Barnes L, Boyle P, Bennett DA. The neuropathology of older persons with and without dementia from community versus clinic cohorts. J Alzheimers Dis 2009;18:691-701.

[58] Schneider JA, Arvanitakis Z, Bang W, Bennett DA. Mixed brain pathologies account for most dementia cases in community-dwelling older persons. Neurology 2007;69:2197-204.

[59] White L. Brain lesions at autopsy in older Japanese-American men as related to cognitive impairment and dementia in the final years of life: a summary report from the Honolulu-Asia aging study. J Alzheimers Dis 2009; 18:713-25.

[60] Launer LJ, Hughes T, Yu B, Masaki K, Petrovitch H, Ross GW, et al Lowering midlife levels of systolic blood pressure as a public health strategy to reduce late-life dementia: perspective from the Honolulu Heart Program/Honolulu Asia Aging Study. Hypertension 2010; 55:1352-9. 